\title{
ADENOID CYSTIC CARCINOMA OF THE BREAST
}

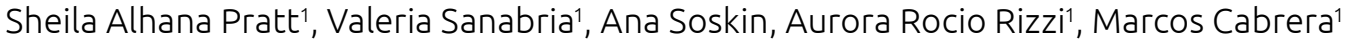

${ }^{1}$ Hospital Nacional de Itaugua - Paraguay.

Introduction: Adenoid cystic adenoid carcinoma (ACC) of the breast is a rare variant that occurs in large series only in less than $1 \%$ of the patients. It has favorable prognosis and morphological resemblance to tumors originating in the salivary glands. Histopathological diagnosis is based on a mixture of proliferating glands, which correspond to the adenoid component and the replication of the basal membrane in the form of cylinders established by the pseudoglandular component. It usually occurs in adult women. Its form of clinical presentation is a painless breast nodule, located in the retroareolar region, without compromise of skin or nipple secretion, of small size and circumscribed limits. Treatment of ACC is not protocolized, although it is accepted that conservative surgery is applicable in most cases. Our goal is to publicize the clinical case of a patient treated in our service and to show some clinical and histopathological aspects of this same rare pathology. Case report: 63-year-old patient, with no history of cancer in the family, menarche at 16 years of age, three full births, 24 months of lactation, menopause at 46 years of age, no hormone replacement therapy. Hypertensive and diabetic, a right breast nodule was self-detected six months before; slow and painless growth that then fistulizes the skin and is accompanied by serohematic secretion of a gelatinous consistency. On physical examination, a $7 \mathrm{~cm}$ nodule is felt in lower quadrants, showing elastic consistency and poorly defined edges. No adenopathies in the armpits or neck. Sectional biopsy performed in another service. Pathological anatomy: differential diagnosis between Cystic Hypersecretory Carcinoma and Cystic Secretory Hyperplasia. With the diagnosis of ACC of the right breast, T2NOMx stage IIa. Mammography: breasts type $b$, at the junction of lower quadrants of the right breast, dense delimited mass measuring $5 \times 4 \mathrm{~cm}$, no retraction or skin edema. BIRADS V. Ecograph: A mass located in $\mathrm{H6}$ to $3 \mathrm{~cm}$ of the nipple with well delimited edges, with heterogeneous characteristics with liquid and solid areas of $5.71 \mathrm{~cm} \mathrm{x} 4.06 \mathrm{~cm}$. BIRADS V. Extensive resection and sentinel node biopsy is performed. Histopathological result revealed ACC G1 of $5.5 \mathrm{~cm}$ with no associated in situ carcinoma. No vascular plungers or perineural invasion are observed. Free surgical limits. pT3 Nx.Mx. Immunohistochemistry: RE-; RP-; HER2-;ki67<14\%. Our case showed a neoplastic proliferation consisting of two types of cavity formation; true glandular lights (adenoid component) and pseudo lumens that produce basal membrane material (cylinder component) with eosinophilic basal membrane material, the adenoid component with basophilic mucin and surrounded by myoepithelial cells. Three negative lymph nodes metastasis. Radiation therapy was decided as an adjuvant treatment. 